\title{
Applications of the Dual Porosity Theory to Irregularly Shaped Porous Materials
}

\author{
François-Xavier Bécot, Luc Jaouen \\ Matelys - Acoustique \& Vibrations, 20/24 rue Robert Desnos, 69120 Vaulx-en-Velin, France. \\ francois-xavier.becot@matelys.com \\ Emmanuel Gourdon \\ Laboratoire des Sciences de l'Habitat, DGCB URA CNRS 1652, Ecole Nationale des Travaux Publics de l'Etat, \\ Membre de l’Université de Lyon, rue Maurice Audin, 69518 Vaulx-en-Velin Cedex, France
}

Invited paper based on a presentation at the ICA 2007 in Madrid

\begin{abstract}
Summary
Non planar acoustic materials may be used in building and environmental acoustics to achieve a significant absorption at low frequencies. Examples of these materials are anechoic wedges or advanced design noise barriers. The shape of these materials is mainly based on empirical knowledge because a fine numerical modeling (e.g. FEM, BEM) requires large computational costs. Therefore, the optimisation of the general form and of the material used to realise these absorbing systems is limited. The purpose of this paper is to propose an original alternative to these limitations. The work basis relies on the theory for the acoustics of multi-scale porous materials, and in particular on double porosity materials, which has been initiated by Olny and Boutin (J. Acoust. Soc. Am. 2003, 114(1)). It is shown in this paper that this theory could be successfully applied to the modeling of non planar sound absorbing materials. Examples are given for multi-layer systems involving perforated panels, material samples having an irregular surface and anechoic wedges. The discussion is based on comparisons between analytical simulations and measurements.
\end{abstract}

PACS no. 43.55.Ev, 43.55.Pe

\section{Introduction}

Controlling the sound at low frequencies is one of the main challenges which has to be faced in Building, Transport and Industry. With a view to design robust passive sound absorbing systems, it has been shown recently that the concept of double porosity could be applied to increase the acoustic absorption at low frequencies for a given range of materials [1]. However, this principle has been applied so far to a limited number of cases.

The present paper proposes to illustrate the double porosity concept on three different sound absorbing systems. The main objective is to show that this theory is found to be a powerful tool to predict and to understand the physical phenomena involved in sound absorbing systems which present non-planar surfaces. The direct application of this work is the design of innovative sound absorbents with optimised geometry and properties.

First, the acoustical behaviour of a multi-layer system consisting of a perforated mineral material backed with a screen and an air gap is examined. This first example is also used to introduce the parameters used in the double

Received 7 May 2008,

accepted 8 August 2008. porosity theory. Second, the acoustic absorption of a material presenting an irregular surface made from perforations having various sizes and deepnesses is considered. Finally, predictions of the sound absorption coefficient of wedges typically used in anechoic rooms are compared to measurements and discussed.

\section{Some elements about the dual porosity theory}

Interested readers are referred to the founding publications for a full theoretical description of the underlying theory. In particular, the authors would like to recommend publications from Olny [2] and Olny and Boutin [1] which discuss the acoustic dissipation mechanisms involved in a medium having two scales of porosity. These developments have been based on initial works by Boutin and Auriault $[3,4,5,6]$ who treated the more general cases of quasi-static and dynamic regimes. All these works are based on the homogenisation method for periodic structures (HSP) as introduced by Sanchez-Palencia [7]. Some elements of the dual porosity principle for acoustic purposes as used in the present work are given here. In order to be complete, one should also mention the research effort 
in the domain of geophysics which has recently produced a number of inspiring papers, e.g. [8, 9, 10, 11].

\subsection{Media with a single porosity}

The materials considered in the present paper comprise two phases: a solid phase, corresponding to the squeleton of the porous medium, which is saturated by the fluid phase, generally air at rest in acoustics. The wavelength of the sound in the surrounding air $\lambda_{0}$ is assumed to be large compared to the size of the observed sample, having a characteristic size $L$. If the characteristic size of the micro-pores $l_{m}$ inside the material is small compared to $L$ or $\lambda_{0}$, the material may be considered as a porous medium with a single scale of porosity $\phi_{m}$. In that sense, the material may be accurately represented by considering a microscopic representative elementary volume which results from the superposition of two homogeneous phases, a fluid phase and a solid phase. This representation comes back to Biot $[12,13]$ and has been extensively used and implemented based on the book of Allard [14].

Submitted to an airborne sound excitation, the two phases interact via viscous, inertial, thermal and possibly structural effects depending on the nature and the morphology of the material and on the frequency. In the low frequency range, the viscous forces dominate the inertial forces. At higher frequencies, viscous forces decrease compared to inertial forces. The limit between these two behaviours is estimated by the so-called viscous characteristic frequency, noted $\omega_{v}$. A similar description can be made for the thermal dissipation mechanisms. Below the thermal characteristic frequency, noted $\omega_{t}$, the fluid motion is mainly isothermal and above $\omega_{t}$, transformations are adiabatic. On top of these two dissipation mechanisms, structural dissipation mechanisms arise when the material skeleton is deformed under the sound wave loading. When this occurs, the displacements in the solid and the fluid phases are strongly coupled and a supplementary dissipation has to be accounted for. An estimation of the frequency above which the two phases can be considered as decoupled is given in [15]. For a material which lays on an acoustically rigid termination and submitted to an airborne sound excitation, viscous dissipation usually dominates the thermal and structural dissipation on the audible frequency range (see for instance Figures 8-10 in [16]).

\subsection{Media with two scales of porosity}

Consider now the situation where the material has some additional air pockets, called meso-pores, with a characteristic size $l_{p}$, which is intermediate between the scale of observation $L$ and the characteristic size of the micropores $l_{m}$. For a double porosity material based on a fibrous medium (see Figure 1), this latter size $l_{m}$ would correspond to the average distance between the fibers. These air inclusions may originate from air blowing during the manufacturing process or perforations done within the finished material. As a result, the nature of the air flow and the



Figure 1. Schematic representation of the different characteristic lengths considered for the representation of single porosity or double porosity media. Left figure: perforated rockwool, right figure: picture of the fiber microscopic network.

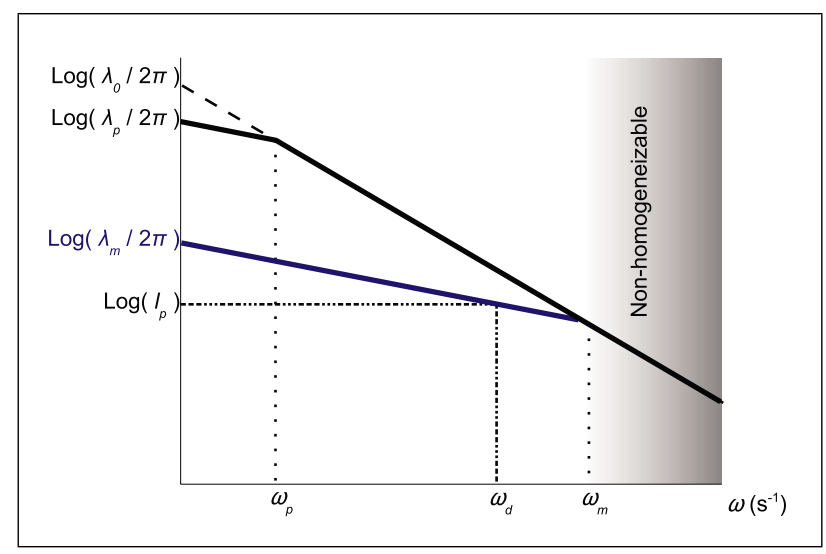

Figure 2. Schematic representation of the different domains for the nature of sound propagation in a dual porosity medium. Represented here is the case of high contrast of permeability between the pores and the meso-pores. Only viscous effects are considered here.

dissipation mechanisms inside the material will be modified according to the relative size of $l_{m}$ and $l_{p}$. The separation of scale implies that two different sound wavelengths should be considered in the networks of micro-pores and of meso-pores respectively. To fully describe these phenomena, separate characteristic frequencies shall be considered: $\omega_{v p}$ and $\omega_{t p}$ will refer to meso-pores and $\omega_{v m}$ and $\omega_{t m}$ will refer to the micro-pores.

The Figure 2 schematically illustrates, as represented in Figure $3 b$ in [1], the different sound wavelengths as functions of the viscous characteristic frequencies in the case of a large separation of scales between the two pore networks. Only viscous dissipation is represented here. In this case, the ratio $l_{p} / l_{m}$ is typically comprised between 10 and $10^{3}$ and a high contrast of permeability between the two networks of pores is observed. The flow in the microporous medium remains viscous on the entire homogenisable frequency range, while the flow in the meso-pores is successively viscous and inertial. Moreover, there exists a frequency at which the wavelength $\lambda_{m}$ in the micro-porous 
medium becomes in the same order of magnitude than the characteristic size of the air pockets $l_{p}$.

As a result, variations of the fluid motion observed at the mesoscopic scale are also perceptible at the microscopic scale. This yields an additional dissipation mechanisms which is interpreted as a pressure diffusion effect between the two networks of pores [1]. This effect reaches a maximum around the so-called diffusion frequency and is located in a limited region of the micro-porous medium close to the interface with the meso-pores. The strong pressure gradient, and thus the large energy dissipation in this region has been illustrated in [1] using analytical expressions and in $[17,18]$ using a variable mesh of finite elements. At the scale of observation, these phenomena corresponds to an increased sound absorption which generally occurs in the low frequency range due to the involved characteristic sizes.

For the principle of double porosity could be advantageously applied, the material should present a high value of the static air flow resistivity and a low value of the high frequency limit of the tortuosity [2]. Typically, fibrous materials having a high density fulfil these criteria. The level and frequency position of the maximum sound absorption is governed by the value of the meso-porosity $\phi_{p}$ which is defined as the ratio between the volume occupied by the meso-pores to the total volume of material and, to a minor extent, by the actual characteristic size $l_{p}$ and the shape of the meso-pores. The influence of these parameters has been numerically verified in $[18,19]$ for a plane wave sound field and experimentally studied in [19, 20, 21] for a diffuse sound field. Recently, double porosity materials used for the control of the sound radiation of a coupled plate cavity sytem have been studied numerically in [21] and experimentally in [22]. Ongoing research aims now at studying the influence of other sound fields or of the pore shapes.

\subsection{Principles of modeling and input data}

The principle of modeling is common to all the configurations presented in this paper. The presence of the two networks of pores implies to use one model to represent the dissipation in the meso-pores and one model to represent the dissipation in the micro-pores. At this stage of the modeling procedure, the two networks of pores are considered independently and may be represented by different dissipation models. On the one hand, the meso-porous domain is modeled as if the micro-porous part were impervious and on the other hand, the micro-porous domain is considered as homogeneous and isotropic. The coupling of the two networks consists in expressing the dynamic permeability and the dynamic bulk modulus of the double porous medium as functions of the same quantities given for the two sub-domains [1].

A large variety of models may be used to represent the dissipation in the porous domains. In this paper, viscothermal effects may be described using the single parameter model described in [23] and modified by [24] or using the five parameter model of $[25,26]$. In addition for the meso-porous domain, the expression of the dissipation can be derived analytically for parallel cylindrical pores as described in [1] from the expressions of [15]. For other geometries, the analogous perforation radius which gives the same meso-porosity may be computed to allow the use of these analytical expressions. Complementary modifications needed to adapt specific coupling conditions with multi-layer components or to specific geometries may also be considered ; they are described below in the description of the tested samples.

The question of the availability of the input data arises now. The geometry of the perforations may be directly measured or deduced from the cutting tool (see section 4). The material parameters are required for each of the components of the multi-layer systems. Each material constituting the layers of the system has been characterized independently. The characterization method used relies on the direct measurements of the open porosity $\phi$ [27], the static air flow resistivity $\sigma$ [28], the dynamic density and the dynamic bulk modulus of the material, these two last properties being obtained from standing wave tube measurements [29]. Parameters related to the material morphology which allow to reproduce the visco-inertial and thermal dissipations effects according to [23, 24, 26, 25, 30] are then deduced from these measurements using the corresponding analytical expressions [29, 31, 32]. These additional parameters are namely the high frequency limit of the tortuosity $\alpha_{\infty}$, the viscous and thermal characteristic lengths, respectively $\Lambda$ and $\Lambda^{\prime}$, and the static thermal permeability $k_{0}^{\prime}$. However, for the materials studied here, the thermal dissipation is negligible compared to viscous dissipation and $k_{0}^{\prime}$ parameter has been omitted.

In the following, three examples of double porosity materials are discussed: first a perforated, multi-layer material, second a material having perforations of irregular depths and third, a material having a non planar surface. The model used and the parameter values for each layer of each example are compiled in Table I and are discussed in the following. For the sake of simplicity, parameters values were rounded. One could also note that for the two last foams reported in this table, the criterion of $\Lambda^{\prime} \approx 2 \Lambda$ is verified. For the materials studied here, dissipation was mainly visco-inertial and only five parameter models $[26,25]$ were used.

\section{Porous perforated panel in a multi-layer system}

This section examines two situations where the double porosity medium is comprised in a multi-layer system. The base configuration is the following: a mineral foam, perforated all the way through the thickness, is coupled to a screen on its rear side, these two layers being backed by a rigidly terminated air plenum (see Figure 3 ). Two systems are then considered depending on the shape and the size of the perforations. The first system presents irregular circular perforations and the second one has regular perforations of square cross-section. The systems are submitted 
Table I. Acoustical models and corresponding parameters for the materials used in the paper. DBM: Delany-Bazley-Miki model [23, 24], JCA: Johnson-Champoux-Allard model [26, 25].

\begin{tabular}{llcccccc}
\hline Material & Model & $\begin{array}{c}\sigma \\
\mathrm{k} \mathrm{N} . \mathrm{s} \cdot \mathrm{m}^{-4}\end{array}$ & $\begin{array}{c}\phi \\
-\end{array}$ & $\begin{array}{c}\alpha_{\infty} \\
-\end{array}$ & $\begin{array}{c}\Lambda \\
\mu \mathrm{m}\end{array}$ & $\begin{array}{c}\Lambda^{\prime} \\
\mu \mathrm{m}\end{array}$ & $\begin{array}{c}k_{0}^{\prime} \\
10^{-10} \mathrm{~m}^{2}\end{array}$ \\
\hline Mineral panel (section 3) & DBM & 2300 & & & & & \\
Screen 1 (section 3) & JCA & 2000 & 0.99 & 1.80 & 1.4 & 1.4 & \\
Screen 2 (section 3) & JCA & 1500 & 0.99 & 2.00 & 2.2 & 2.2 & \\
Foam (section 4) & JCA & 130 & 0.98 & 1.00 & 50.0 & 100.0 & 10 \\
Melamine foam (section 5) & JCA & 10 & 0.99 & 1.00 & 100 & 200 & 150 \\
\hline
\end{tabular}

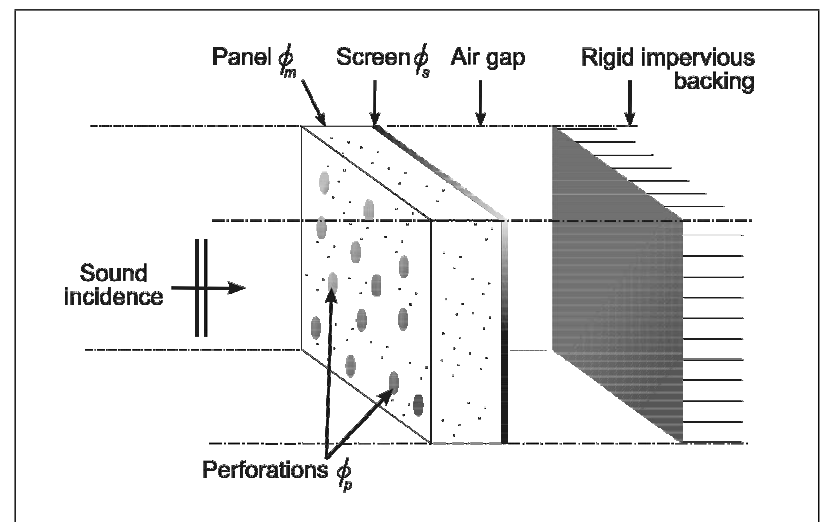

Figure 3. Scheme of the three-layer system composed by a perforated panel, a screen and an air gap for normal plane wave incidence.

to plane waves and to a diffuse sound field. Those systems are commonly used in Buildings as sound absorbing ceilings.

\subsection{Acoustical characterisation of the system com- ponents}

The panel having cylindrical perforations consists of a $8.5 \mathrm{~mm}$ thick porous mineral panel backed with a screen of thickness around $0.1 \mathrm{~mm}$ (screen 1 in Table I) and an $100 \mathrm{~mm}$ air gap. Some holes have an irregular cross section but, from the modeling point of view, all holes will be considered to have the same diameter equal to the mean characteristic size of the perforations, namely $3 \mathrm{~mm}$. The total meso-porosity of the panel $\phi_{p}$ is $9 \%$. The panel with regular square perforations of $90 \mathrm{~mm}^{2}$ section area has a thickness of $12.5 \mathrm{~mm}$ and is backed by $0.2 \mathrm{~mm}$ screen (screen 2 in Table I) and a $100 \mathrm{~mm}$ air gap. In this case, the mesoporosity value, $16 \%$, has been calculated from the actual value of the perforation sizes. From the modeling point of view, the value of an analogous circular perforation radius has been calculated to give the same value of the mesoporosity.

For the two systems considered here, the dissipation inside the micro-porous domain was found to be correctly reproduced using the empirical relations derived by Delany-Bazley [23] and further modified by Miki [24]. These expressions involve only one parameter: the static air flow resistivity $\sigma_{m}$. Visco-thermal dissipation inside the meso-porous domain was correctly reproduced using the Johnson-Champoux-Allard model [25, 26] whose parameters are calculated from the perforation size or the analogous perforation radius and the perforation rate $\phi_{p}$. An example of such calculations can be found in chapter 5 of Allard's book [14]. Finally, the visco-thermal dissipation inside the screens was modelled using a JohnsonChampoux-Allard model [26, 25]. Values of the acoustical parameters obtained after the characterization procedure as described in Section 2.3, for each layer, are given in Table I.

\subsection{Modeling of the multi-layer systems}

A first model, called here after "single" porosity model, can be considered by neglecting the internal porosity of the panel material. For this model, the visco-thermal dissipation as described in $[25,26]$ is calculated using a corrected value of $\alpha_{\infty}$ to account for the added mass effect and the radiation damping at each perforation end. This correction is based on the radiation of infinitely baffled circular piston. Note that more advanced correction terms were also proposed recently in [33]. In a second model, the internal porosity of the panel and the additional dissipative effects resulting from the coupling between the micro-scale of porosity and the scale of the perforations are accounted for. The method used here is the analytic approach based on the theory presented in [1]. This second model is called here after "double" porosity model. These two models assume that the porous medium has a rigid and motionless solid phase.

Since the screen is glued onto the perforated panel and is very thin compared to the acoustic wavelength, the values used in the models for the porosity and for the air flow resistivity have to be modified compared to the measured values. When considering the panel-screen system, the porosity $\phi_{s}$ is changed to $\phi_{s} \times \phi_{p}$ and the resistivity $\sigma_{s}$ is replaced by $\sigma_{s} / \phi_{p}$.

The indicator of the acoustic performance discussed here is the sound absorption coefficient. For plane wave under normal incidence, the calculation is straightforward using standard expressions which can be found for instance in [14]. Diffuse sound field conditions are reproduced using the procedure as proposed in [34]: the material response is calculated by adding up the material responses for a range of incidences comprised between 0 and 78 degrees. To improve the accuracy of the summation, angles are distributed according to a Gauss-Legendre quadrature using a minimum of 20 points. 


\subsection{Comparisons between measurements and simu- lations}

For the two systems, simulation results are compared for a plane wave excitation and a diffuse sound field. For plane wave incidence, the sound absorption coefficients are measured in an impedance tube of $46 \mathrm{~mm}$ diameter. Rigourously speaking, the entire reproductible pattern for each of the two systems tested here is assumed to have a size of $600 \times 600 \mathrm{~mm}^{2}$ cross section. Therefore, circular samples of diameter $46 \mathrm{~mm}$ may not contain an elementary reproduction of the entire pattern. The absorption efficient measured in these conditions may vary from one sample to another and should be examined with care. To overcome this, a larger sample having a total size of approximately $12 \mathrm{~m}^{2}$ was made, the surface of which contains several elementary patterns. The complete sample was tested in diffuse sound field conditions according to ISO 354 [35]. Measurements were carried out by an independant laboratory for the third octave bands of frequency from $100 \mathrm{~Hz}$ band to the $4000 \mathrm{~Hz}$. Simulation results were obtained using a narrow frequency step $(1 \mathrm{~Hz})$. In all configurations, the presence of the air plenum yields an interference pattern whose period is proportional to the depth of the air gap.

Results using the single and double porosity models described previously are reported in Figure 4, for the material having circular perforations. For plane wave incidence (top graphs), the two models give values of the absorption coefficient which are very similar at very low frequencies, under $200 \mathrm{~Hz}$. At these frequencies, the dissipative effects in the microporous domain are negligible because of the very large wavelength compared to the characteristic dimension of this microprous part. At higher frequencies and up to $2000 \mathrm{~Hz}$, the single porosity model gives predictions which are lower than those obtained using the double porosity model, which means that some dissipation mechanisms are not included in this model. In the low frequency range, single porosity results are closer to the measurements, while the double porosity model tends to give better predictions of the measured values at higher frequencies. Thus, as the frequency increases, the interaction between the two network of pores becomes more significant and the double porosity model seems more suited to predict these mechanisms compared to the single porosity model which only considers the panel perforations.

For the diffuse field conditions, the double porosity model gives predictions which correspond well with the measured values while the single porosity model fail to predict correctly these values. The single porosity model clearly underestimates the measured absorption coefficient which means that some dissipation mechanisms are not included in this model. The size of the tested samples appear to be crucial to show experimentally the double porosity effects and to validate the double porosity model. In addition, the presence of oblique incidences may lead to an increase of the coupling between the two pore networks. For these incidences could enter the micro-porous domain through the walls of the meso-pores and thus create an ap-

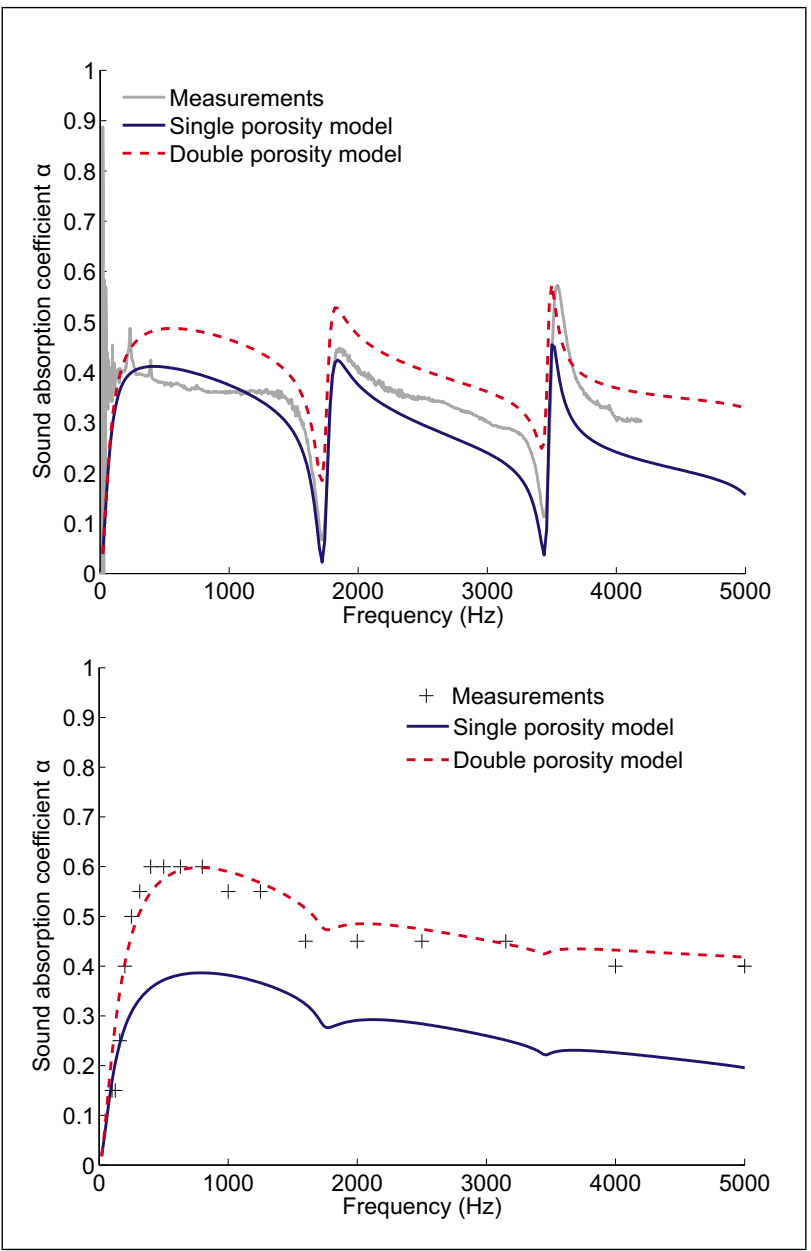

Figure 4. Comparison between sound absorption coefficients simulated using a single or a double porosity model and measured. System: panel with circular perforation backed by a $100 \mathrm{~mm}$ air gap. Top: impedance tube conditions; Bottom: diffuse field conditions.

parent coupling between the perforations and the panel. This effect is however difficult to quantify.

Figure 5 presents the measured and simulated sound absorption coefficients for the materials having square perforations. In the case of plane wave incidence (top figure), the two models give very similar predictions below 2 $000 \mathrm{~Hz}$. Beyond this frequency, none of the models manage to estimate correctly the measured absorption coefficients. However, the double porosity model gives predictions which are closer to the measurements and in particular in the vincinity of the second dip of absorption around $3500 \mathrm{~Hz}$. This tends to prove that the dissipation is better estimated in the double porosity model. At frequencies above $3500 \mathrm{~Hz}$, predictions using this model are in a better agreement with the measured values compared to those obtained using the single porosity model. As for the material with circular perforations, the size of the samples tested in this configuration (in a $46 \mathrm{~mm}$ impedance tube) does not allow to illustrate fully the double porosity effects.

Results for diffuse field conditions (bottom graphs) show larger deviations between the results obtained us- 
ing the single and the double porosity models. As for the other system, results from the double porosity model follow closely the measurements in third octave bands while results from the single porosity model clearly underestimates the measured values. These results allow to validate the double porosity model proposed here to reproduce the coupling between the square perforations and the microporous part of the material.

Finally, results presented here tend to show the dual porosity principle could be successfully applied to predict the sound absorption of perforated micro-porous material. It also appears that for this material, the geometry of the perforations plays a minor role. Finally, the deviations observed between the measurements and the simulations for impedance tube conditions, seem to be related to the representativity of the tested samples. Increasing the size of the sample allows to have a more complete picture of the elementary periodic pattern of the material. In this case, measurements show a larger effect of the coupling between the two pore networks and model simulations tend to confirm the importance of including double porosity effects to improve the prediction accuracy.

\section{Material with perforations of various shapes}

The second series of double porosity media studied in this paper is made from a polymer foam perforated with holes having different radii and thicknesses. The originality of these configurations compared to those studied in Sgard et al. [18] lies in the fact that perforations are not compact nor centered. It is shown here that the dual porosity theory could be applied successfully to media which present an intricate surface.

\subsection{System characteristics and modeling}

The base material has been chosen for its high static air flow resistivity, high open porosity and low tortuosity which are the double porosity key parameters to observe noticeable dissipative effects. Dissipation occuring in the foam are found to be correctly reproduced using the Johnson-Champoux-Allard [26, 25] model. The values of the model parameters, reported in Table I, were obtained using the characterization technique described by Olny et al. in [29, 31, 32].

The tested samples comprise a series of holes whose geometries are shown in the Figure 6. Tested samples are all $30 \mathrm{~mm}$ thick and are laid on an acoustically rigid termination. Perforations with two different diameters have been made: $6.6 \mathrm{~mm}$ and $15 \mathrm{~mm}$. In addition, perforations could have three different depths: $10 \mathrm{~mm}, 20 \mathrm{~mm}$ or $30 \mathrm{~mm}$. As a result, the meso-porosity may take on three different values along the thickness for the various samples (see Figure 7). For our tested samples, the number of holes and the successive values of the meso-porosity, expressed in $\%$ and rounded, from the sound incidence to the rigid backing are respectively: sample (i), 1 hole and $\phi_{p}=2-2-2$; sample (ii),

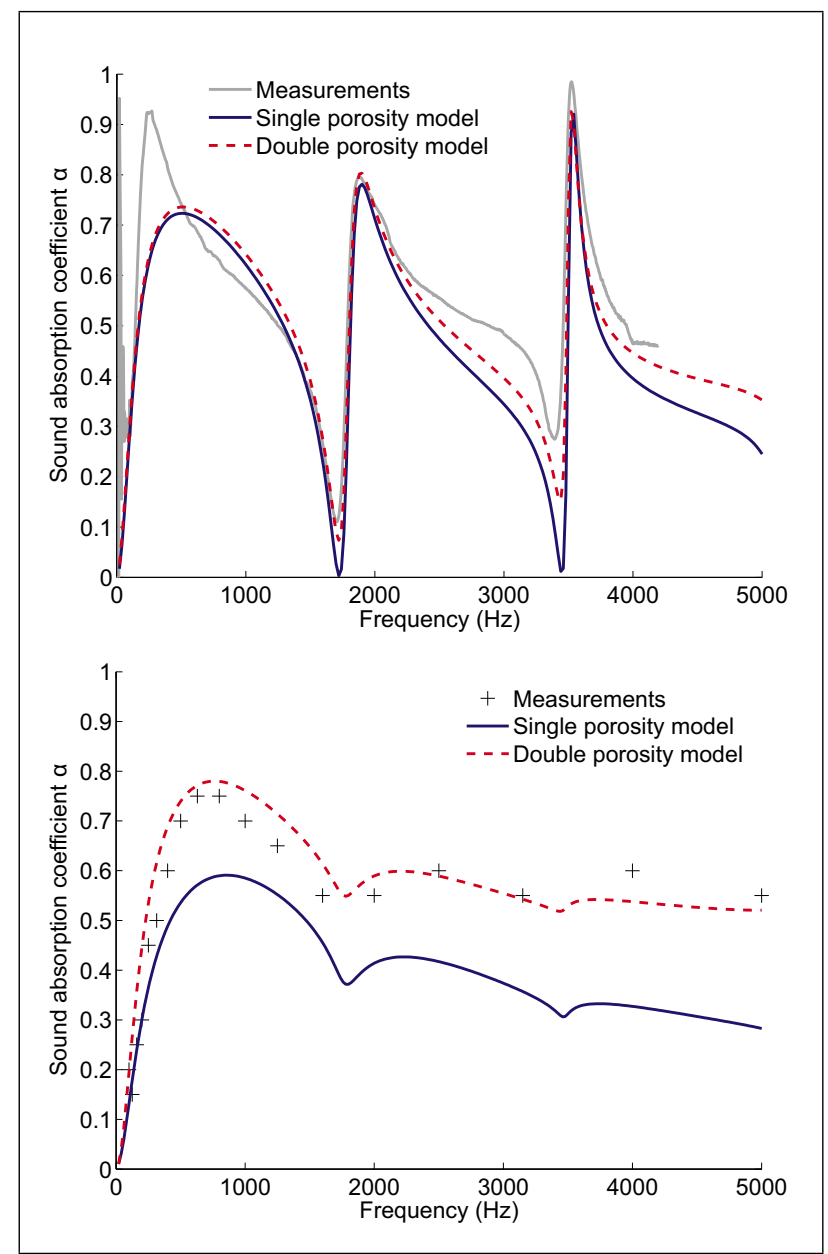

Figure 5. Comparison between sound absorption coefficients simulated using a single or a double porosity model and measured. System: panel with square perforations backed by a $100 \mathrm{~mm}$ air gap. Top: impedance tube conditions; Bottom: diffuse field conditions.

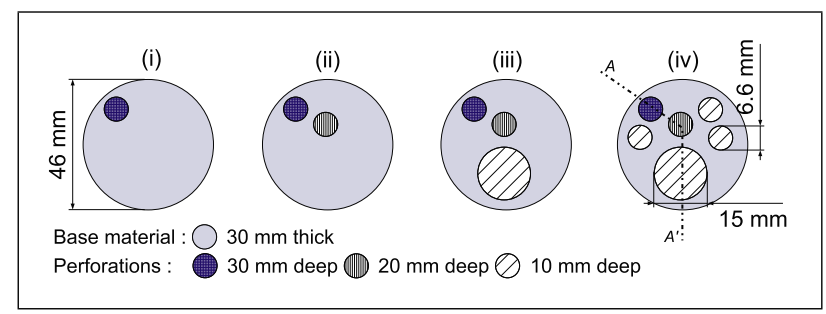

Figure 6. Geometry of the perforated material samples. View of the faces toward sound incidence.

2 holes and $\phi_{p}=4-4-2$; sample (iii), 3 holes and $\phi_{p}=14-4-$ 2 ; sample (iv), 6 holes and $\phi_{p}=21-4-2$.

On the modeling point of view, each of the three layers is represented by a medium having a circular perforation with a radius which gives the same value as the actual meso-porosity. Moreover, the geometric parameter $D_{0}$ introduced by Olny in [1] and the characteristic diffusion length $\Lambda_{D}$ have to be modified as done by Sgard in [18] to cope with the fact that the actual perforations are not realised all through the material. 


\subsection{Measured and simulated absorption coefficients}

The sound absorption coefficients were measured in the same $46 \mathrm{~mm}$ diameter impedance tube used for the previous example. For all configurations, the elementary perforation patterns of the tested samples correspond closely to the one which was modeled. Thus any limitations related to the representativity of the sample size are avoided.

In Figure 8, a close correspondence between the measured (thin lines) and the predicted values (thick lines) is observed on the entire frequency range and for all configurations from (i) to (iv). Besides the frequency range from around $1500 \mathrm{~Hz}$ to $2700 \mathrm{~Hz}$ where structural effects appear for the single porosity sample ("SP") and for sample (i), both the level and the evolution of the dissipation mechanisms are correctly captured. It should be emphasised that the section profile of these samples, in particular for sample (iv), corresponds to a very irregular surface as illustrated in Figure 7.

Therefore, these results show that the actual distribution of the perforations inside the material is not significant, even with fairly large perforation sizes. They also prove that an accurate modeling of the sound absorption from irregular surface materials could be achieved by using the double porosity concept. This kind of materials may be found in Buildings or in advanced noise barriers design.

\section{Materials with non planar surfaces}

The final examples given in this paper are two wedged shaped materials used for sound absorption at very low frequencies in anechoic or semi-anechoic laboratory rooms. The efficiency of this type of systems is usually evaluated in terms of the "critical frequency" which corresponds to the frequency beyond which the absorption coefficient is larger than $99 \%$. The objective is to show that modeling of this type of geometry, which usually requires large computational means using finite element methods, can be efficiently achieved using analytical calculations based on the dual porosity theory. Compared to the work presented in [36], different models are used here to represent the dissipation inside the micro-porous part of the double porosity medium.

\subsection{Description of the samples and modeling}

The anechoic wedges considered here are made of a standard melamine foam which has been characterised using the method described in [29]. It comprises a heel and a wedge whose respective dimensions are indicated by $H$ and $L$ in the following. In addition, the anechoic wedges are backed by an air plenum of thickness indicated by $P$. The first anechoic wedge has the following dimensions $L=900 \mathrm{~mm}, H=200 \mathrm{~mm}, P=110 \mathrm{~mm}$ and is covered with perforated metal sheet. The presence of the metal covering modifies the nature of the dissipation mechanisms in a limited frequency range as discussed below. The second wedge has no covering and has the following dimensions:

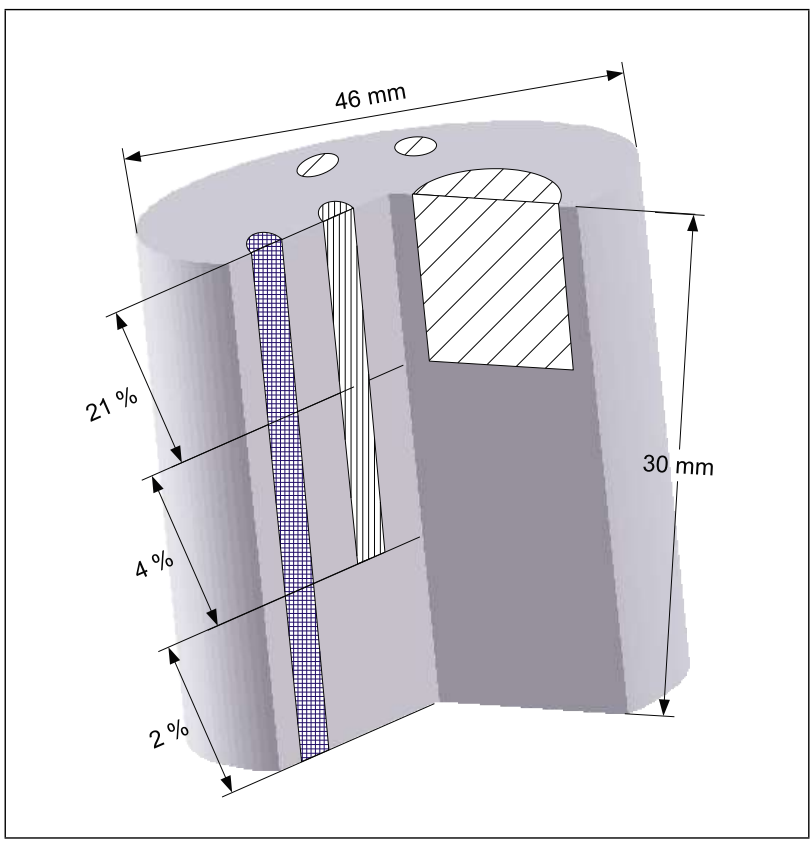

Figure 7. In depth geometry of sample (iv) along the line $A-A^{\prime}$ (see Figure 6).

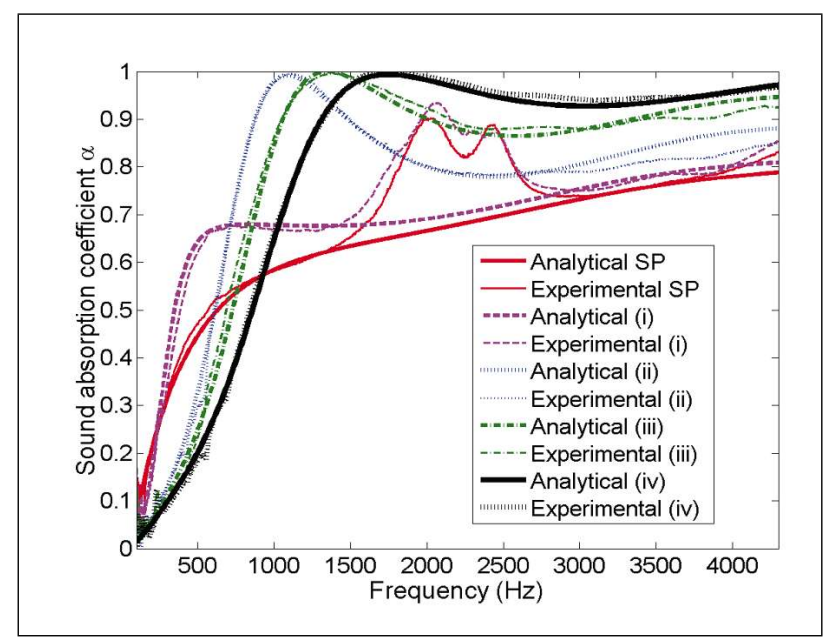

Figure 8. Absorption coefficients measured and simulated of double porosity materials with non-centred perforations. $\mathrm{SP}=$ single porosity, material without perforation. Plane wave normal incidence conditions.

$L=630 \mathrm{~mm}, H=380 \mathrm{~mm}, P=100 \mathrm{~mm}$. Both wedges have a heel of squared cross section of $300 \times 300 \mathrm{~mm}^{2}$.

The complete wedge is modelled using a stratification of the inclined part of the wedge. It has been shown previously [36] that the dual porosity theory could be applied successfully to this type of geometry provided that a sufficient number of layers are accounted for. The mesoporosity of each layer is computed according to the actual wedge geometry. Each layer is then considered as a double porosity material having a meso-perforation of cylindrical shape. The analogous perforation radius is calculated to give the actual value of the meso-porosity. An example of the stratification procedure is given in Figure 9. The grey area shows the micro-porous part of the double porosity 
model. Since we examine the plane wave condition, this pattern could be infinitely reproduced in adjacent cells. By doing so (light grey area in the figure), the shape of an approached wedge appears more clearly.

The visco-thermal dissipation in the heel and in the micro-porous part of the double porosity material is reproduced using the Johnson-Champoux-Allard model [26, 25]. This model, referred to as "rigid frame" below, assumes that the skeleton of the porous material is rigid and motionless. In some cases and certain frequency ranges, the inertial effects may be significant. In this case, one of the alternate "equivalent fluid" models should be used. These models, described for instance in [37, 38, 16, 39], consider either that the skeleton manifests no stiffness, "limp" model, or that the skeleton is infinitely rigid but can move as a whole, "rigid body" model [16]. These models have been proved to yield similar calculations for material having a porosity close to one [16]. Therefore, only the assumption of an infinitely soft skeleton was used in the present calculations.

\subsection{Model convergence}

Absorption coefficients of the complete systems were measured in the large impedance tube of $600 \times 600 \mathrm{~mm}^{2}$ square cross section. The cut-off frequency for these tube dimensions is $285 \mathrm{~Hz}$. However, by using an adapted excitation signal and by measuring the sound pressure on the axis of the tube, it was checked that accurate measurements could be performed up to the second transverse mode [2]. Thus, the valid frequency range spans from $20 \mathrm{~Hz}$ to $500 \mathrm{~Hz}$. The lateral dimensions of one wedge is $300 \times 300 \mathrm{~mm}^{2}$. Therefore, four wedges were placed in staggered rows to fill in the tube section which allow a good representativity of the elementary periodic pattern compared to the modeled one. The sound pressure was measured at four points located on the axis of the tube, the closest position to the wedge being at a minimum distance of $0.1 \mathrm{~m}$ away from the peaks.

An example of calculations using an increasing number of layers is shown in Figure 10. One can observe that the 6 layers' model gives predictions which follow closely the measured values of sound absorption coefficients. Since working with a larger number of layers is computationally costless, the calculations shown in the following use a 11 layers' model. For the inclined part of the wedge, discretised using 10 layers, a rigid frame model was applied. For the heel, which is represented using a single layer, either a "limp" model or a "rigid frame" model was used.

\subsection{Wedge performance predictions}

Results for the wedge with no metal cover are presented in Figure 11. It is observed that accounting for a possible frame motion of the heel improves significantly the prediction of the critical frequency compared to a "rigid motionless" model. The critical frequency is predicted within a $15 \%$ error using this model. The overall behaviour of the absorption coefficient at frequencies below $150 \mathrm{~Hz}$ is also correctly captured. However, the exact prediction of

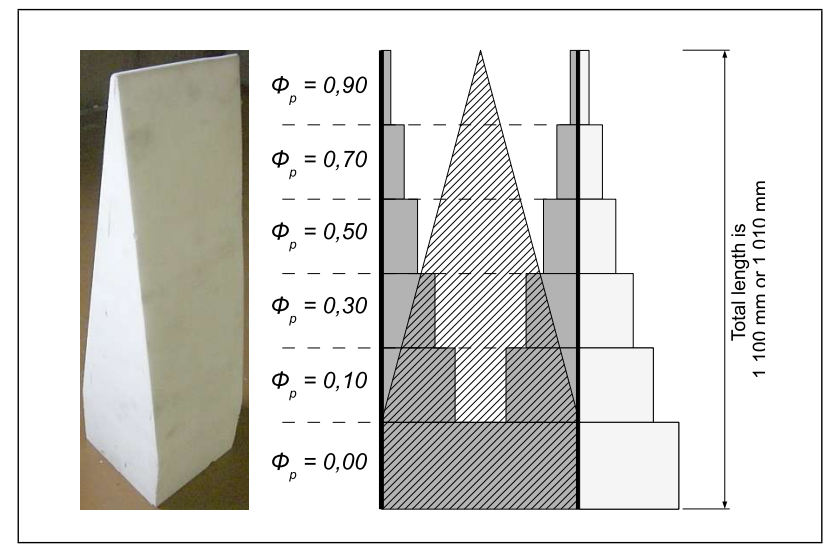

Figure 9. Picture of the actual anechoic wedge and illustration of the corresponding double porosity model using 6 layers. Dashed area: actual geometry of the wedge, black vertical lines: tube walls, grey area: micro-porous part, ligth grey area: continutation of the periodic pattern.

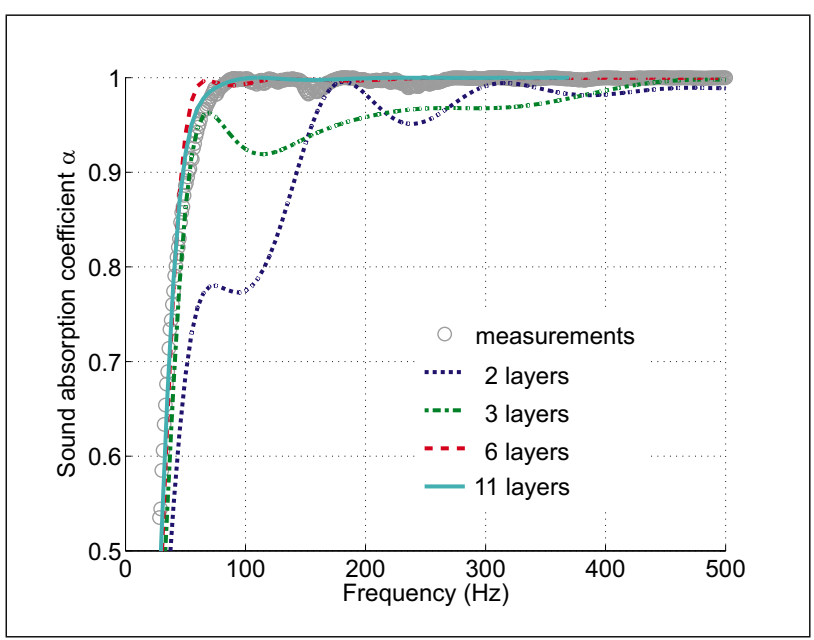

Figure 10. Sound absorption coefficients calculated for an increasing number of layers (numbers include the heel). Dimensions: $L=900 \mathrm{~mm}, H=200 \mathrm{~mm}, P=110 \mathrm{~mm}$, perforated metallic covering.

this value depends strongly on the exact boundary conditions between the material and the tube surfaces which are difficult to quantify. Accounting for elastic effects in the successive double porosity layers is expected to increase further the accuracy of the predictions. This is the subject of ongoing research.

Figure 12 compares the measured values and the predictions in the case the wedge is covered with a metal sheet whose perforation rate is approximately $25 \%$ with hole sizes of 2,5 or $10 \mathrm{~mm}$. It is thus considered as acoustically transparent and is not represented in the model. The effect of the metal cover mainly consists in constraining the porous material and hence, in reducing the contributions of the inertial and structural effects in the observed frequency range. Actually, it is observed in this figure that the "rigid motionless" porous model predicts the critical frequency with a satisfactory accuracy (about $1 \%$ error) contrary to the "limp" model (100\% error). Moreover, the 




Figure 11. Absorption coefficients of anechoic wedges measured and simulated in impedance tube conditions. Dimensions: $L=630 \mathrm{~mm}, H=380 \mathrm{~mm}, P=100 \mathrm{~mm}$.



Figure 12. Absorption coefficients of anechoic wedges measured and simulated in impedance tube conditions. Dimensions: $L=900 \mathrm{~mm}, H=200 \mathrm{~mm}, P=110 \mathrm{~mm}$, perforated metallic covering.

general behaviour of the absorption below the cut-off frequency is also correctly reproduced.

\section{Discussion}

In this paper, the double porosity theory based on the homogenisation technique as derived in $[1,5,6]$ has been successfully applied to predict the acoustical behaviour of porous structures having complicated surfaces. Various materials have been used from low resistive (melamine foam) to high resistive foam (mineral foam).

Multi-layer systems have been examined which allow to discuss the coupling of a double porosity medium with other media. Sound absorption coefficients of regularly or irregularly perforated materials have been correctly reproduced. This has underlined the importance of testing samples which reproduce an entire elementary periodic pattern of the perforated material in order to obtain accurate pre- diction of the measured sound absorption. It was shown that, for the materials tested here, the shape of the perforations played a minor role in the prediction of the sound absorption coefficient. This property allowed to build an analytical model of typical wedges used in anechoic rooms which gives satisfactory predictions as long as the mounting conditions are correctly accounted for.

The main limitations of the application of this theory to a larger number of materials concerned the correspondance between the actual geometry of the meso-domain and the one which can be modeled analytically. So far, only cylindrical perforations may be correctly included. Deriving the diffusion function for other geometries could overcome this and is the subject of ongoing work.

Besides these restrictions, the various examples discussed above proved that this theory is a powerful approach to understand the physical mechanisms involved in materials which were so far modelled using computationally demanding methods. With the analytical tools presented here, the acoustical behaviour of complicated structures under plane waves or diffuse field excitation could be modeled and optimised. Works are currently in progress to develop further this theory to account for more elaborate coupling configurations.

\section{Acknowledgement}

The authors are grateful to Xavier Olny and Claude Boutin for the long and fruitful discussions related to the acoustics of multi-scale porous materials.

The authors would like to thank Dick Botteldooren, Editor-in-Chief of Acta Acustica united with Acustica, for the invitation to propose an extended version of this work which was first presented at ICA 2007 conference [40].

\section{References}

[1] X. Olny, C. Boutin: Acoustic wave propagation in double porosity media. J. Acoust. Soc. Am. 114 (2003) 73-89.

[2] X. Olny: Absorption acoustique des milieux poreux à simple et double porosité - modélisation et validation expérimentale - transl. "acoustic absorption of porous media with single and double porosity - modeling and experimental validation". PhD Dissertation. ENTPE - INSA-Lyon, 1999.

[3] J.-L. Auriault, C. Boutin: Deformable media with double porosity - quasi-statics. i : Coupling effects. Transport in porous media 7 (1992) 63-82.

[4] J.-L. Auriault, C. Boutin: Deformable media with double porosity - quasi-statics. ii : Memory effects. Transport in porous media 10 (1993) 153-159.

[5] J.-L. Auriault, C. Boutin: Deformable media with double porosity - iii : Acoustics. Transport in porous media 14 (1994) 143-162.

[6] C. Boutin, P. Royer, J.-L. Auriault: Acoustic absorption of porous surfacing with dual porosity. Int. J. Solids Structures 35 (1998) 4709-4737.

[7] E. Sanchez-Palencia: Non-homogeneous media and vibration theory, lecture notes in physics. J. Ehlers et al. Springer-Verlag, Berlin, 1980, 398. 
[8] J. G. Berryman, H. F. Wang: Elastic wave propagation and attenuation in a double-porosity dual-permeability medium. Int. J. Rock Mech. Min. Sci. 37 (2000) 63-78.

[9] S. R. Pride, J. G. Berryman: Linear dynamics of doubleporosity dual-permeability materials. I. Governing equations and acoustic attenuation. Physical Review E 68 (2003).

[10] S. R. Pride, J. G. Berryman: Linear dynamics of doubleporosity dual-permeability materials. II. Fluid transport equations. Physical Review E 68 (2003).

[11] S. R. Pride, J. G. Berryman, J. M. Harris: Seismic attenuation due to wave-induced flow. J. Geophys. Res. 109 (2004).

[12] M. Biot: Theory of propagation of elastic waves in a fluidsaturated porous solid. I. Low frequency range. J. Acoust. Soc. Am. 28 (1956) 168-178.

[13] M. Biot: Theory of propagation of elastic waves in a fluidsaturated porous solid. II. Higher frequency range. J. Acoust. Soc. Am. 28 (1956) 179-191.

[14] J.-F. Allard: Propagation of sound in porous media. modeling sound absorbing materials. Elsevier applied sciences, 1993.

[15] C. Zwikker, C. Kosten: Sound absorbing materials. Elseveir, New York, 1949.

[16] F.-X. Bécot, F. Sgard: On the use of poroelastic materials for the control of the sound radiated by a cavity backed plate. J. Acoust. Soc. Am. 120 (2006) 2055-2066.

[17] F. Castel: Modélisation numérique de matériaux poreux hétérogènes - application à l'absorption basse fréquence. PhD Dissertation. INSA-Lyon - Sherbrooke university, 2005.

[18] F. Sgard, X. Olny, N. Atalla, F. Castel: On the use of perforations to improve the sound absorption of porous materials. Applied Acoustics 66 (2005) 625-651.

[19] N. Atalla, C. K. Amédin, R. Panneton, F. Sgard: Etude numérique et expérimentale de l'absorption acoustique et de la transparence acoustique des matériaux poreux hétérogènes en basses fréquences dans le but d'identifier des solutions à fort potentiel d'applicabilité. Tech. Rept. R-278, IRSST Montréal, Canada, 2001.

[20] N. Atalla, C. K. Amédin, Y. Atalla, R. Panneton, F. Sgard: Développement de nouveaux matériaux absorbants à hautes performances acoustiques pour diminuer le bruit en basses fréquences. Tech. Rept. R-370, IRSST Montréal, Canada, 2004.

[21] F.-X. Bécot, F. Sgard: Advanced heterogeneous materials for noise and vibration control. Proceedings of SAPEM 2005, Symposium on the Acoustics of Poro-Elastic Materials, Lyon, France, 2005.

[22] F.-X. Bécot, F. Sgard, N. Amirouche: Noise control optimisation of machinery enclosures : theory, materials and experiments. Proceedings of Noise at Work, Lille, France, 2007.
[23] M. E. Delany, E. N. Bazley: Acoustical properties of fibrous absorbent materials. Applied Acoustics 3 (1970) 105-116.

[24] Y. Miki: Acoustical properties of porous materials - modifications of Delany-Bazley models. J. Acoust. Soc. Jpn 11 (1990) 19-24.

[25] Y. Champoux, J.-F. Allard: Dynamic tortuosity and bulk modulus in air-saturated porous media. J. Appl. Phys. 70 (1991) 1975-1979.

[26] D. L. Johnson, J. Koplik, R. Dashen: Theory of dynamic permeability and tortuosity in fluid-saturated porous media. J. Fluid Mech. 176 (1987) 379-402.

[27] L. L. Beranek: Acoustic impedance of porous materials. J. Acoust. Soc. Am. 13 (1942) 248-260.

[28] A. C-522: Standard test method for airflow resistance of acoustical materials. American Society for Testing and Materials (2003).

[29] X. Olny, R. Panneton, J. Tran-van: An indirect acoustical method for determining intrinsic parameters of porous materials. Poromechanics II, Proceedings of the $2^{\text {nde }}$ BIOT conference, 2002.

[30] D. Lafarge, P. Lemarinier, J.-F. Allard, V. Tarnow: Dynamic compressibility of air in porous structures at audible frequencies. J. Acoust. Soc. Am. 102 (1997) 1995-2006.

[31] R. Panneton, X. Olny: Acoustical determination of the parameters governing viscous dissipation in porous media. J. Acoust. Soc. Am. 119 (2006) 2027-2040.

[32] X. Olny, R. Panneton: Acoustical determination of the parameters governing thermal dissipation in porous media. J. Acoust. Soc. Am. 123 (2008) 814-824.

[33] N. Atalla, F. Sgard: Modelling of perforated plates and screens using rigid frame porous models. J. Sound Vib. 303 (2007) 195-208.

[34] L. L. Beranek: Noise reduction. McGraw-Hill, NewYork, 1971. Chapitre 13.

[35] N. E. I. 354: Acoustics - measurement of sound absorption in a reverberation room. International Organization for Standardization (2003).

[36] X. Olny: Acoustical properties of multiscales absorbing porous materials. Proceedings of SAPEM (Lyon, France), 2005.

[37] H.-Y. Lai: Modeling of acoustical properties of limp fibrous materials. Dissertation. PhD thesis, Purdue University, USA, 1997.

[38] J. Tran-Van, X. Olny, F. Sgard: Effect of porous media transverse isotropy on sound transmission properties of double wall systems. Proceedings of Forum Acusticum 2002, Sevilla, Spain, 2002.

[39] R. Panneton: Comments on the limp frame equivalent fluid model for porous media. J. Acoust. Soc. Am. 122 (2007) EL217-EL222.

[40] F.-X. Bécot, L. Jaouen, E. Gourdon: Modeling the absorption of non-planar acoustic materials. Proceedings of $19^{\text {th }}$ International Conference on Acoustics, Madrid, Spain, 2007. 